\title{
Hyperspectral Imaging and Spectral-Spatial Classification For Cancer Detection
}

\author{
Baowei Fei ${ }^{1,2,3,4, *}$, Hamed Akbari ${ }^{1}$, Luma V. Halig ${ }^{1}$ \\ ${ }^{1}$ Department of Radiology and Imaging Sciences, Emory University School of Medicine \\ ${ }^{2}$ Department of Biomedical Engineering, Emory University and Georgia Institute of Technology \\ ${ }^{3}$ Department of Mathematics and Computer Sciences, Emory University \\ ${ }^{4}$ Winship Cancer Institute of Emory University \\ Atlanta, GA, U.S.A
}

\begin{abstract}
Hyperspectral imaging is an emerging modality for medical applications. Its spectroscopic data can be utilized to noninvasively detect cancer. In this study, an advanced image processing and classification method is proposed to analyze hyperspectral image data for prostate cancer detection. Least squares support vector machines (LS-SVMs) were developed and evaluated for classifying hyperspectral data in order to enhance the detection of cancer tissue. The method was used to detect prostate cancer in tumor-bearing mice. Spatially resolved images were created to highlight the differences of the reflectance properties of cancer versus those of normal tissue. Preliminary results with 11 mice show that the hyperspectral imaging and classification method was able to reliably detect prostate tumors in the animal model. This study may lead to advances in the optical diagnosis of cancer using hyperspectral imaging.
\end{abstract}

Keyword: Hyperspectral imaging, spectral-spatial classification, support vector machine, prostate cancer detection

\section{INTRODUCTION}

Hyperspectral imaging (HSI) is a spin-off from remote sensing technology developed by NASA for space exploration and earth observation [1]. Hyperspectral imaging can extend human vision to infrared and near-infrared wavelength regions. In fact, the imaging system produces several narrow band images at different wavelengths. Compared to conventional color cameras and other filter-based imaging systems, this system produces full neighboring spectral data with spectral and spatial information [2]. In medical applications, hyperspectral image data provide a powerful tool for noninvasive tissue analysis. Hyperspectral imaging has been used to provide quantitative data regarding tissue oxygen saturation in patients with peripheral vascular disease [3], to detect ischemic regions of the intestine during surgery [4], to predict and follow healing in foot ulcers of diabetic patients [5], and to diagnose hemorrhagic shock [6].

Hyperspectral imaging has been used to discriminate between cancerous and normal tissue [7]. However, this method is limited to the visible wavelength range and requires

\footnotetext{
* Corresponding author: bfei@emory.edu,www.feilab.org

The 5th IEEE International Congress on Image and Signal

Processing (CISP 2012) and the 5th International Conference on BioMedical Engineering and Informatics (BMEI 2012), Chongqing, China, October 16-18, 2012.
}

the injection of fluorescent material. Hyperspectral imaging has also been evaluated for use in the cytologic diagnosis of cancer cells [8]. It has been reported that hyperspectral imaging is also used to detect gastric tumors in human subjects [9]. The spectral signatures of the gastric cancer and non-cancerous stomach tissue were created in infrared wavelengths. An HSI system with the spectral range of 500-600 $\mathrm{nm}$ has demonstrated its surgical application in breast cancer animal models [10].

The spectral information contained in hyperspectral image data allows characterization, identification, and classification of different types of tissue. Various image processing methods have been developed in an attempt to resolve the hyperspectral data classification problem. The support vector machine (SVM) is one of the successful approaches to multispectral data classification [11-14]. The properties of SVMs make them well-suited to tackle this classification problem as they can: 1) efficiently manage large input spaces; 2) deal with noisy samples in a robust way; and 3) produce sparse solutions, i.e., the decision boundary is expressed as a function of a subset of training samples.

In this research, we performed in vivo hyperspectral imaging experiments and proposed an SVM method for hyperspectral image classification of prostate tumor tissue. We identify spectral signatures of both normal and cancerous tissue in animals. The details of the experiments and methods are described in the following sections.

\section{MATERIALS AND METHODS}

\section{A. Hyperspectral Imaging System}

To capture the hyperspectral image data, a CRi camera systems (Caliper, Hopkinton, MA) was used to acquire images from animals. The system is a light-tight apparatus that uses a Cermax-type 300 Watt Xenon light source. This provides $5600^{\circ} \mathrm{K}$ that spans the electromagnetic spectrum from 500 $950 \mathrm{~nm}$. The CCD is a 16-bit, high-resolution, scientific-grade imaging sensor. Four, fiber-optic, adjustable illuminator arms yield an even light distribution to the subject. The light radiates from the excitation source and then illuminates the sample. Reflect lights pass through the camera lens to the solid-state liquid crystal tuning element and finally to the CCD. The excitation and emission filter sliders hold two, 50-mm diameter, long pass filters. The long pass filters remove the 
band light especially from the excitation source. These filters are color coded to indicate the wavelength they represent. The field of view (length $\times$ width) is from $3.4 \times 2.5 \mathrm{~cm}$ to $10.2 \times 7.6$ $\mathrm{cm}$ with variable zoom. The resolution is from 25 to $75 \mu \mathrm{m}$ based on the zoom lens position. The fluence rate, i.e., the flow of photons over a set angle, is from 4 to $20 \mathrm{~mW} / \mathrm{cm}^{2}$ based on the light position. The scan time is from $5 \mathrm{sec}$ to $1 \mathrm{~min}$.

\section{B. Animal Tumor Model}

An androgen-dependent prostate tumor xenograft model CWR22 was originally derived from a primary human prostatic carcinoma $[15,16]$. The frozen CWR22 cancer cells were thawed in $37^{\circ} \mathrm{C}$ water, washed with tissue culture medium (RPMI 1640, Hyclone Laboratories, Inc., Logan, UT) with $20 \%$ calf serum (Hyclone Laboratories, Inc., Logan, UT), and were then filtered through a single layer of Nitex with 100 $\mu \mathrm{m}$ porosity (Tetko, Inc., Briarcliff Manor, NY). The cells were suspended in Matrigel (BD Biosciences, Bedford, MA) with a volume at least equal to that of the cell volume. The suspension was draw into 1.0-cc syringes with 19-gauge needles for a $0.2-\mathrm{mL}$ volume per injection. Athymic nude mice 4-8 weeks old were housed under controlled conditions (12 h dark and light cycles; $20-24^{\circ} \mathrm{C}$ temperature) and with freely reachable sterilized mouse chow [17]. A 12.5-mg sustained-release testosterone pellet (Innovative Research of America, Sarasota, FL) was implanted in each nude mouse one week before cell injection. Each animal was subcutaneously given an injection of cells suspension on the other side of the implanted testosterone. The animal experiments were approved by our Institutional Animal Care and Use Committee (IACUC) and conformed to the guidelines of the National Institutes of Health for the care and use of laboratory animals.

In this study, the hyperspectral imaging experiment was performed on 11 male nude mice. Nine mice had human prostate tumors growing on the flank. During image acquisition, each mouse was anesthetized by i.p. injection of a ketamine and xylazine mixture at a dose of ketamine at 95 $\mathrm{mg} / \mathrm{kg}$ and xylazine at $5 \mathrm{mg} / \mathrm{kg}$ body weight.

\section{Support Vector Machine (SVM) for Image Classification}

In our study, least squares SVM (LS-SVM) is proposed to classify the hyperspectral data. LS-SVM has previously been applied to other applications [18]. This LS-SVM method allows us to interpret and design learning algorithms geometrically in the kernel space that is nonlinearly related to the input space, thus combining statistics and geometry in an effective way. In LS-SVMs, the algorithm attempts to identify a ridge regression for classification using binary targets. Therefore, LS-SVM overcomes some of the disadvantages of classical SVM that attempts to identify a large margin for classification. In LS-SVMs, equality constraints are considered for the classification problem with a formulation in the least squares sense. As a result the solution follows directly from solving a set of linear equations, instead of quadratic programming.
We first segment the tumor on one group of hyperspectral images for LS-SVM training. For a new group of hyperspectral images, the trained LS-SVM is used to perform the automatic classification of tumors on the new hyperspectral images. We have input vectors of 251 elements in 2-nm spectral resolution images. The output of the trained LS-SVM is the classification result of normal and cancer tissue on the image.

\section{Classification Evaluation}

The performance of the automatic classification was evaluated with respect to the gold-standard maps created manually by a medical doctor. Sensitivity and specificity were used as statistical measures of the performance of the binary classification method [19-21]. Sensitivity measures the proportion of actual positives which are correctly identified as positive, i.e. the percentage of tumor pixels which are correctly identified as tumor tissue. When a pixel was not detected as a tumor pixel, the detection was considered as a false negative if the pixel was indeed a tumor pixel in the manually created map. When a pixel was detected as a tumor tissue, the detection was a false positive if the pixel was not tumor tissue. Specificity measures the proportion of negatives which are correctly identified, i.e., the percentage of healthy tissue correctly identified as not having cancer.

\section{RESULTS}

Fig. 1 shows the hyperspectral images of a tumor-bearing mouse. The hyperspectral image cube consists of twodimensional images at each narrow band wavelength and onedimensional spectra at each pixel. As shown on the graph (Fig.1B), the spectra of the normal and tumor tissue are different. The spectral information was used by the SVM-based classification to differentiate normal and cancer tissue on the hyperspectral images. For the 11 mice, the sensitivity and specificity were $92.8 \pm 2.0 \%$ and $96.9 \pm 1.3 \%$, respectively.

\section{CONCLUSION}

We developed a hyperspectral image classification method for prostate cancer detection in an animal model. A least-squares support vector machine classifier was used to classify cancer tissue in animals. Our preliminary study has demonstrated the feasibility of using hyperspectral imaging and quantitative analysis methods for prostate cancer detection. Hyperspectral imaging offers a potential, noninvasive tool for surgeons to inspect and assess a large area of tissue without taking tissue sample for pathology examination. This method allows continuous evaluation of suspicious cancer tissue without interrupting surgery and could, therefore, be used as a virtual biopsy tool.

\section{ACKNOWLEDGMENT}

This research is supported in part by NIH grant R01CA156775 (PI: Fei), Coulter Translational Research Grant (PIs: Fei and $\mathrm{Hu}$ ), Georgia Cancer Coalition Distinguished Clinicians and Scientists Award (PI: Fei), Emory Molecular and Translational Imaging Center (NIH P50CA128301), SPORE in Head and Neck Cancer (NIH P50CA128613), and 
Atlanta Clinical and Translational Science Institute (ACTSI) that is supported by PHS Grant UL1 RR025008 from the Clinical and Translational Science Award program.

\section{REFERENCES}

[1] A. F. H. Goetz, "Three decades of hyperspectral remote sensing of the Earth: A personal view," Remote Sensing of Environment, vol. 113, pp. S5-S16, Sep 2009.

[2] h. Akbari, et al., "Hyperspectral Image Segmentation and its Application in Abdominal Surgery," International Journal of Functional Informatics and Personalised Medicine, vol. 2, pp. 201-216, 2009.

[3] D. C. Kellicut, et al., "Emerging Technology: Hyperspectral Imaging," Perspectives in Vascular Surgery and Endovascular Therapy, vol. 16, pp. 53-57, 2004.

[4] H. Akbari, et al., "Detection and analysis of the intestinal ischemia using visible and invisible hyperspectral imaging," IEEE Trans Biomed.Eng., vol. 57, pp. 2011-2017, 2010.

[5] L. Khaodhiar, et al., "The use of medical hyperspectral technology to evaluate microcirculatory chaves in diabetic foot ulcers and to predict clinical outcomes," Diabetes Care, vol. 30, pp. 903-910, 2007.

[6] L. C. Cancio, et al., "Hyperspectral imaging: A new approach to the diagnosis of hemorrhagic shock," Journal of Trauma-Injury Infection and Critical Care, vol. 60, pp. 1087-1095, 2006.

[7] M. E. Martin, et al., "Development of an advanced hyperspectral imaging (HSI) system with applications for cancer detection," Annals of Biomedical Engineering, vol. 34, pp. 1061-1068, Jun 2006.

[8] A. M. Siddiqi, et al., "Use of hyperspectral imaging to distinguish normal, precancerous, and cancerous cells," Cancer Cytopathology, vol. 114, pp. 13-21, Feb 252008.

[9] H. Akbari, et al., "Cancer detection using infrared hyperspectral imaging," Cancer Science, vol. 102, pp. 852-857, Apr 2011.

[10] S. V. Panasyuk, et al., "Medical hyperspectral imaging to facilitate residual tumor identification during surgery," Cancer Biology \& Therapy, vol. 6, pp. 439-446, Mar 2007.
[11] G. Camps-Valls and L. Bruzzone, "Kernel-based methods for hyperspectral image classification," IEEE Transactions on Geoscience and Remote Sensing, vol. 43, pp. 1351-1362, 2005.

[12] M. Brown, et al., "Linear spectral mixture models and support vector machines for remote sensing," Geoscience and Remote Sensing, IEEE Transactions on, vol. 38, pp. 2346-2360, 2000.

[13] F. Melgani and L. Bruzzone, "Classification of hyperspectral remote sensing images with support vector machines," IEEE Transactions on Geoscience and Remote Sensing, vol. 42, pp. 1778-1790, 2004.

[14] H. Akbari, et al., "Detection of cancer metastasis using a novel macroscopic hyperspectral method," in Medical Imaging 2012: Biomedical Applications in Molecular, Structural, and Functional Imaging, San Diego, California, USA, 2012, p. 831711.

[15] M. A. Wainstein, et al., "CWR22: androgen-dependent xenograft model derived from a primary human prostatic carcinoma," Cancer Res., vol. 54, pp. 6049-6052, 1994.

[16] H. Wang and B. Fei, "Diffusion-weighted MRI for monitoring tumor response to photodynamic therapy.," J Magn Reson Imaging, vol. 32, pp. 409-17, Aug 2010.

[17] B. Fei, et al., "High-field magnetic resonance imaging of the response of human prostate cancer to Pc 4-based photodynamic therapy in an animal model.," Lasers Surg Med, vol. 39, pp. 723-30, Oct 2007.

[18] Z. Liu, et al., "Automated tongue segmentation in hyperspectral images for medicine," Appl Opt, vol. 46, pp. 8328-34, Dec 12007.

[19] H. S. Wang and B. W. Fei, "A modified fuzzy C-means classification method using a multiscale diffusion filtering scheme," Medical Image Analysis, vol. 13, pp. 193-202, Apr 2009.

[20] X. F. Yang and B. W. Fei, "A multiscale and multiblock fuzzy C-means classification method for brain MR images," Medical Physics, vol. 38, pp. 2879-2891, Jun 2011

[21] K. Bogie, et al., "New technique for real-time interface pressure analysis: getting more out of large image data sets.," J Rehabil Res Dev, vol. 45, pp. 523-35, 2008.
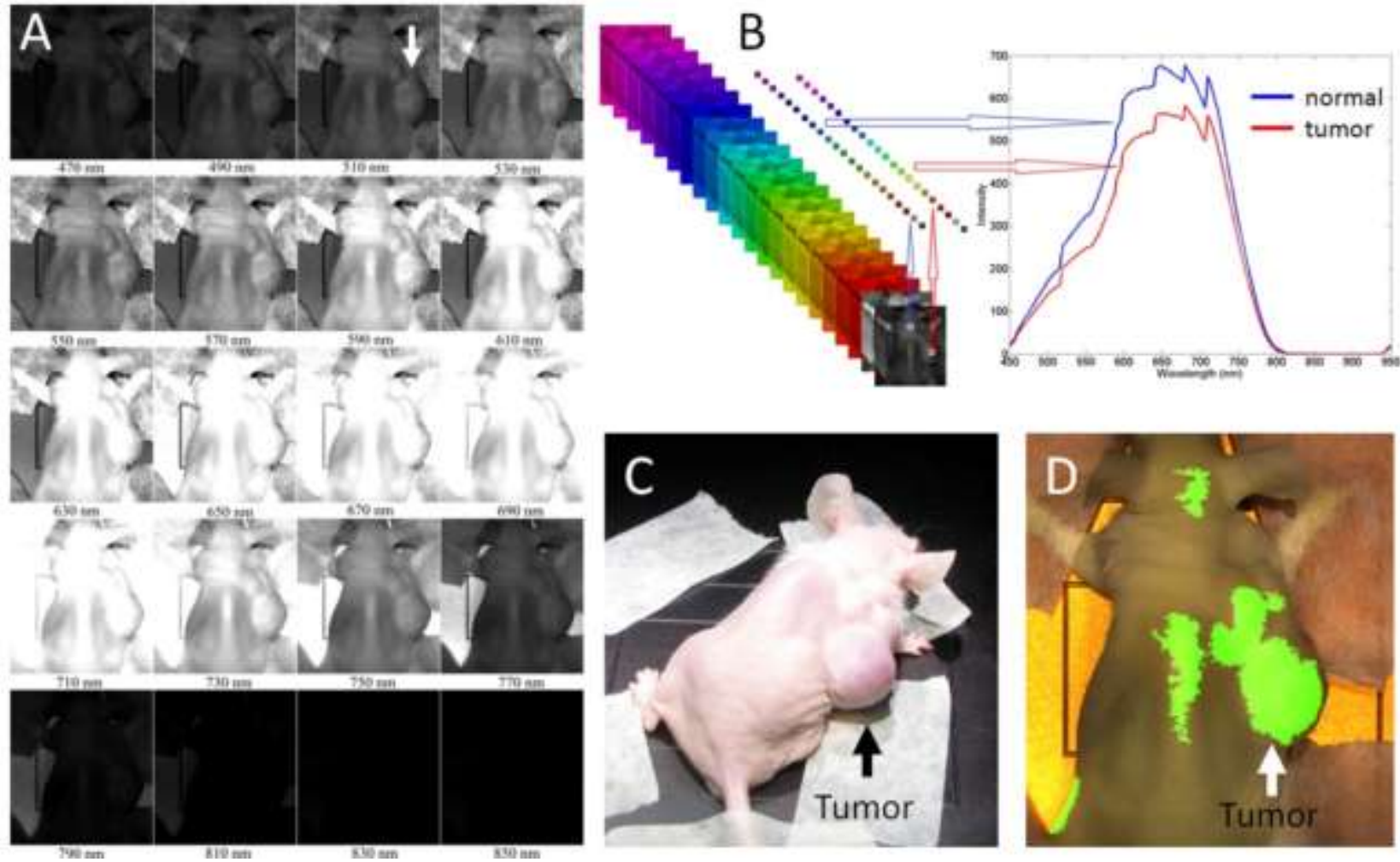

Fig. 1. Hyperspectral imaging of a tumor-bearing mouse. A: HSI images obtained at different wavelengths. B: Schematic view of the HSI cube and the spectral graphs of two, sample pixels, one from cancerous and the other from normal tissue. The graph depicts the normalized reflectance for each wavelength in that pixel. The $\mathrm{X}$ axis shows different wavelengths in nanometers, and the $\mathrm{Y}$ axis shows the normalized reflectance. $C$ : Tumor-bearing mouse. $D$ : Cancer detection results using the LS-SVM pixelwise classification method in the nude mouse where green regions show the tumor. 
Copyright (C) 2012 IEEE. Reprinted from Fei BW, Akbari H, Halig LV, Hyperspectral imaging and spectralspatial classification for cancer detection, The 5th IEEE International Congress on Image and Signal Processing (CISP 2012) and the 5th International Conference on BioMedical Engineering and Informatics (BMEI 2012), Chongqing, China, October 16-18, 2012.

This material is posted here with permission of the IEEE. Internal or personal use of this material is permitted. However, permission to reprint/republish this material for advertising or promotional purposes or for creating new collective works for resale or redistribution must be obtained from the IEEE by writing to pubs-permissions@ieee.org. By choosing to view this document, you agree to all provisions of the copyright laws protecting it. 